\title{
Convective instability in inhomogeneous media: impulse response in the subcritical cylinder wake
}

\author{
C. Marais, ${ }^{1}$ R. Godoy-Diana,, , D. Barkley, ${ }^{2}$ and J.E. Wesfreid ${ }^{1}$ \\ ${ }^{1}$ PMMH, UMR7636 CNRS, ESPCI ParisTech, UPMC, University Denis Diderot, France \\ ${ }^{2}$ Mathematics Institute, University of Warwick, Coventry CV4 7AL United Kingdom
}

(Dated: October 9, 2018)

\begin{abstract}
We study experimentally the impulse response of a cylinder wake below the critical Reynolds number of the Bénard-von Kármán instability. In this subcritical regime, a localized inhomogeneous region of convective instability exists which causes initial perturbations to be transiently amplified. The aim of this work is to quantify the evolution resulting from this convective instability using twodimensional particle image velocimetry in a hydrodynamic tunnel experiment. The velocity fields allow us to describe the evolution of wave packets in terms of two control parameters: the Reynolds number and the magnitude of the imposed perturbation. The temporal evolution of energy exhibits a transient algebraic growth at short times followed by an exponential decay.
\end{abstract}

PACS numbers: 47.20.Ib 47.15.Tr

Keywords: wake instabilities, transient growth, hydrodynamic stability, experiment

\section{INTRODUCTION}

Flow past a circular cylinder is a classic prototype for studying hydrodynamic instabilities and bifurcations in separated flows [1 -7]. Moreover, the academic case of a two-dimensional cylinder wake can be used as a basic model for many real situations, including the flow behind support cables or around an airfoil. When the Reynolds number $\operatorname{Re}=U_{0} D / \nu$ (where $U_{0}$ is the free-stream velocity, $D$ is the cylinder diameter, and $\nu$ is the kinematic viscosity) reaches a particular critical value $\left(\operatorname{Re}_{c} \approx 47\right.$ for an infinitely long cylinder, [1, 2]), a sustained periodic shedding of opposite-signed vortices gives rise to the well-known Bénard-von Kármán vortex street.

The transition to sustained oscillations can be described locally, via wake profiles at different spatial stations, or globally, viewing the $2 \mathrm{D}$ wake as whole. To elaborate further, we first recall the standard distinction between convective and absolute instability in parallel flows illustrated in Figs. 1(a) and 1(b). An instability is convective if a perturbation grows but is simultaneously advected with the flow such that the disturbance decays at any fixed point, as in Fig. 1(a), while it is absolute if the perturbation grows at a fixed spatial position, as in Fig. 1(b) [8 10]. A useful way to distinguish convective from absolute instability is in terms of propagating edge or front velocities: assuming a positive leading-edge velocity $V^{+}$, as is the case here, convective instability corresponds to a positive trailing-edge velocity $V^{-}$, while absolute instability corresponds to a negative trailing-edge velocity [9-13].

Local analysis of wake profiles, i.e. a parallel flow approximation, gives a picture of the transition to sustained oscillations as follows [4, 13, 14]. Above Re $\simeq 5$ there is a local region of convectively unstable flow in the wake.

*Electronic address: ramiro@pmmh.espci.fr

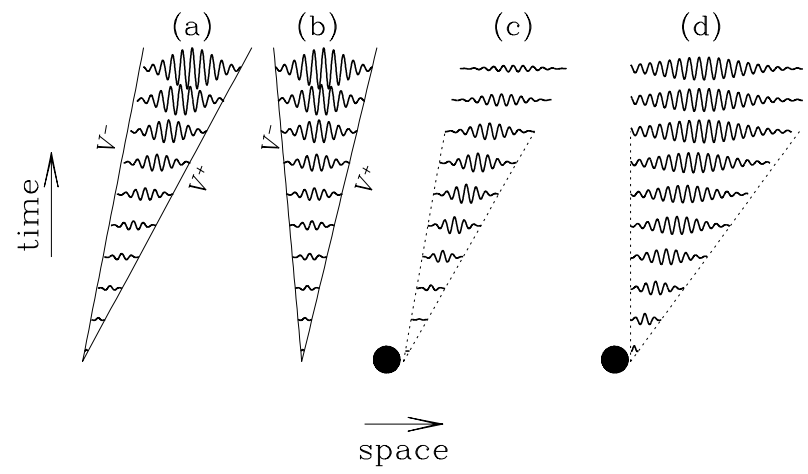

FIG. 1: Sketch of the space-time response of flows to infinitesimal perturbations. (a) and (b) correspond to local analysis, i.e. parallel flows, illustrating the distinction between (a) convective and (b) absolute instabilities. The leading-edge velocity $V^{+}$is positive in both cases. For convective instability, the trailing-edge velocity $V^{-}$is also positive, while for absolute instability it is negative. (c) and (d) correspond to the global wake illustrating the distinction between the (c) subcritical, $\operatorname{Re}<\operatorname{Re}_{c}$, and (d) supercritical, $R e>\operatorname{Re}_{c}$, cases. In the subcritical case, the perturbation reaches a maximum and subsequently decays. In the supercritical case the perturbation continues to grow until it saturates nonlinearly. The edge velocities can be obtained over finite times only in experiment (see Sec. III).

Above $R e \simeq 25$ there is additionally a pocket of absolutely unstable flow within the region of convective instability. Once the pocket of absolutely unstable flow becomes sufficiently large, the flow becomes globally unstable. Globally, however, one does not observe the onset of a locally absolutely unstable region. Instead, one finds that below $\mathrm{Re}_{c}$ the wake responds to perturbations, but only transiently, as perturbations are advected through the systems. This is illustrated in Fig. 1(c). Above $\mathrm{Re}_{c}$, as in Fig. $1(\mathrm{~d})$, perturbations grow and lead to a 
(a)

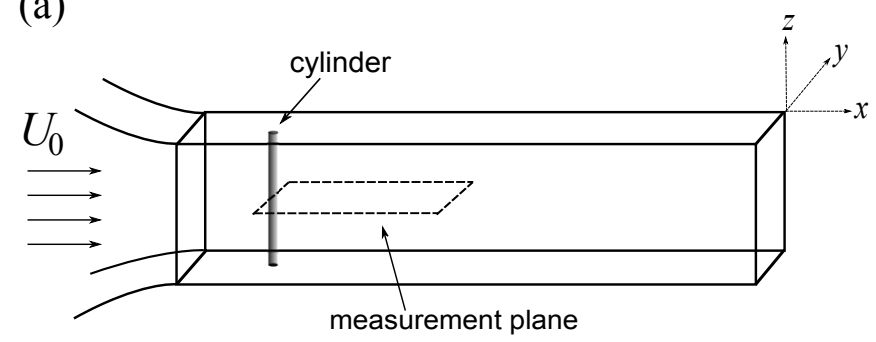

(b)

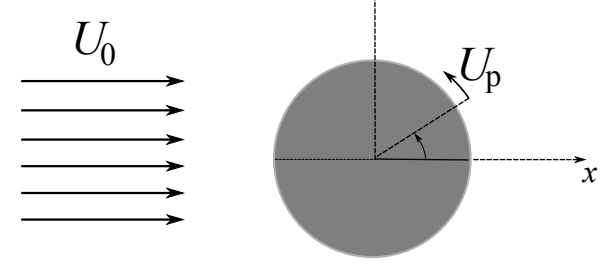

FIG. 2: (a) Schematic diagram of the experimental setup in the hydrodynamic tunnel. (b) Sketch showing a top view of the cylinder during an impulsive rotation of intensity $U_{p}$.

synchronized wake in the formation of what is called a global mode [5, 15]. Hence, even though convective and absolute instability are strictly defined for parallel flows and streamwise period flows [9, 10, 16], inhomogeneous flows, such as flow past a cylinder, may exhibit similar characteristics. The transient response in the subcritical regime has the hallmarks of convective instability while the global instability above $\operatorname{Re}_{c}$ has the hallmarks of absolute instability.

Despite the large body of work on the cylinder wake, only a few experimental studies have examined the wake's subcritical behavior [17] and none have quantitatively characterized the transient dynamics in this regime. The goal of the present paper is therefore to analyze quantitatively the subcritical regime using a well-controlled experiment.

After describing the experimental setup, we focus firstly on the evolution of amplified wave packets and on obtaining the leading-edge, trailing-edge, and group velocities in an experimental setting. The decrease of trailing-edge velocity towards zero when approaching the global instability threshold confirms the transition to an absolute instability. The subcritical behavior is further characterized in terms of the evolution of the maximum amplitude of the wave packet, and its space-time position, as a function of the strength of the perturbation and the distance to the Bénard-von Kármán instability threshold. Finally, to quantify the transient growth phenomenon due to the inhomogeneity of the media, we analyze the temporal evolution of the energy of the perturbation.

\section{EXPERIMENTAL SETUP}

A cylinder of diameter $D=5 \mathrm{~mm}$ is placed in a hydrodynamic tunnel of section $100 \mathrm{~mm} \times 100 \mathrm{~mm}$ (see Fig. 2) with a nearly plug flow in the test section. The boundary layer width in the tunnel walls is of $\sim 10 \mathrm{~mm}$ in the region of interest. The cylinder span is $98 \mathrm{~mm}$ which covers practically the whole height of the tunnel. We use a Cartesian coordinate system, placed in the cylinder center, with the $x$-axis pointing in the flow direction and the $z$-axis running along the cylinder centerline. The cylinder can be put into rotation to provide impulse perturbations to the flow. Measurements are taken in the horizontal mid-plane of the channel.

We define and work with the reduced Reynolds number $\epsilon=\left(\operatorname{Re}-\operatorname{Re}_{c}\right) / \operatorname{Re}_{c}$, corresponding to the distance from the global instability threshold. The critical Reynolds number measured in this experiment is $\operatorname{Re}_{c} \approx 64$, which is larger than the ideal 2D case, primarily due to confinement and blockage effects. We adjust the Reynolds number in the experiment by controlling the flow rate in the tunnel. In the results that follow, $\epsilon$ varies from -0.30 to -0.04 . For this range of Reynolds numbers, the flow remains two-dimensional over nearly the entire cylinder span.

Impulse perturbations consist of applying very short rotary motions to the cylinder. Rotation is controlled by a programmable microstepping electronic module which gives a resolution of $1 / 256$ per full step, allowing for a precise control of the cylinder motion. In the present work, we fix the non-dimensional time interval over which the cylinder is rotated, and use the speed of rotation as the amplitude of the perturbation. More specifically, the perturbation amplitude is defined from the tangential speed of rotation at the cylinder surface $U_{p}$ (see Fig. 21). We consider three perturbation amplitudes given by three values of the non-dimensional rotation speed: $U_{p} / U_{0}=75,100$ and 125 , which we refer to as small, medium and strong, respectively. $U_{0}$ is the measured velocity of the flat profile. The small-amplitude perturbation is the smallest perturbation that produced an observable response in the wake. For all applied perturbations, the cylinder is rotated for a fix dimensionless duration given by $\Delta t / T_{a d v} \approx 0.2$, where $\Delta t$ is the dimensional duration of the perturbation and $T_{a d v}=D / U_{0}$. Note that since $U_{0}$ varies with Reynolds number, both the dimensional tangential speed $U_{p}$ and the dimensional duration $\triangle t$, vary with Reynolds number.

The wave packet generated by the perturbation can be observed qualitatively by visualizing streaklines, as in the work of [17]. Fig. 3 shows this type of visualization compared with snapshots of the cross-stream component of the velocity field obtained by 2D Particle Image Velocimetry (PIV) in the horizontal mid-plane. This highlights a significant difference between the present experiment and previous studies of the subcritical wake response. Velocity field measurements obtained by PIV permit one to study directly the spatial structure of ve- 


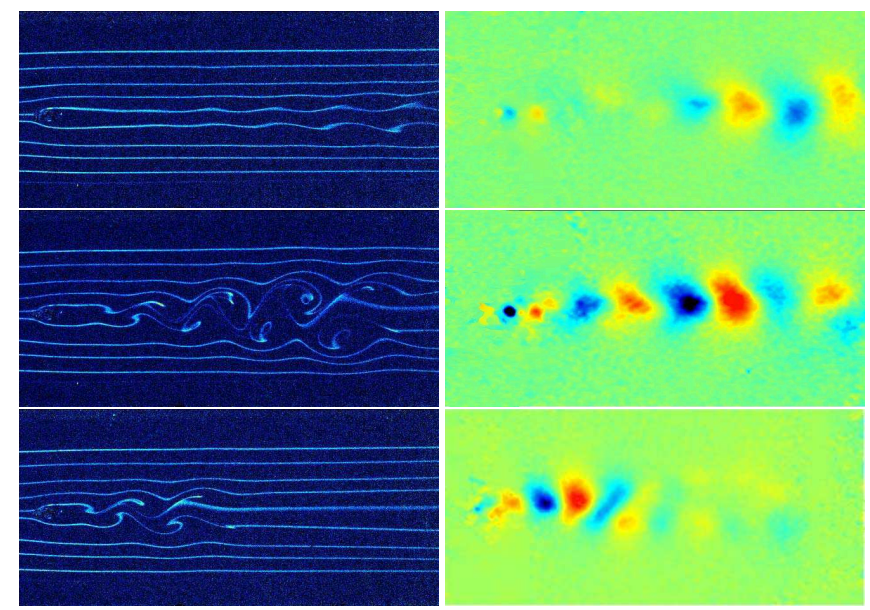

FIG. 3: Visualization of the impulse response at three successive time instants (from bottom to top) in the cylinder wake. Left: streaklines obtained from Fluorescein dye visualization. Right: instantaneous cross stream velocity field obtained from PIV measurements.

locity perturbations as they evolve. The velocity perturbations exhibit a well-defined maximum in the wake. These are impossible to determine from streakline records since the deformation amplitude of injected dye never decays downstream, an artifact caused by mass conservation of the dyed fluid. Therefore, streakline deformation does not give information about the amplitude of the velocity fluctuations $U_{y}$, and thus about the spatial evolution of the impulse response. PIV acquisition and post-processing have been performed using a LaVision system with an ImagerPro 1600 x 1200 CCD camera with a 12-bit dynamic range capable of recording doubleframe pairs of images up to $15 \mathrm{~Hz}$ and a two rod Nd:YAG $(15 \mathrm{~mJ})$ pulsed laser. The time lapse between two frames is set to $20 \mathrm{~ms}$. Finally, additional post-processing and analysis has been carried out with Matlab and the PIVMat Toolbox.

\section{EVOLUTION OF THE WAVE PACKET}

The convective nature of the impulsively perturbed cylinder wake is clearly illustrated in the experimental data shown in Figs. 廿(a) and 4(b). In Fig. 廿(a), crossstream velocity $U_{y}$ profiles, measured at successive times along the wake symmetry axis $y=0$, are stacked up to form a spatio-temporal diagram. The cross-stream velocity on the symmetry axis is the ideal quantity to use to investigate the perturbed flow field because it is everywhere zero for the unperturbed flow. Figure 4(b) is similar to Fig. 4(a) except that the envelope [obtained through a Hilbert transform of $U_{y}$ as illustrated in Fig. [(c)] is plotted.

We measure a number of velocities associated with the space-time evolution of the wave packets. From the Hilbert transform of the cross-stream velocity we are able to extract, at each time instant, the envelope of the wave packet as illustrated in Fig. 4(c). Procedurally, we define the leading and trailing positions as the inflection points of hyperbolic tangent fits to the relevant parts of the envelope at each time. Such fits, together with the associated inflection points, are included in Fig. 4(c). Then we obtain the leading-edge velocity $V^{+}$and the trailingedge velocity $V^{-}$from the collection of fronts extracted from the envelopes at short times $\left(\lesssim 50 T_{a d v}\right)$. Likewise, we obtain the envelope maximum at each time and from these data we define the group velocity $V_{g}$ to be the velocity of envelope maximum, as this gives the speed of the packet as a whole. These velocities are the inverse of the slopes shown in the spatiotemporal diagram of Fig. 世(b). Finally, we define the phase velocity $V_{p}$ as the translation speed of the vortices in the evolving wave packet as seen in Fig. 4(a). Note that all vortices move at essentially identical speed. Moreover, the leading-edge velocity $\mathrm{V}^{+}$, which is effectively determined by the velocity of the first advected vortex released from the cylinder, is the same as the phase velocity to within experimental precision: $V^{+} \simeq V_{p}$

We show now that the edge velocities obtained procedurally through inflections points of the envelop correspond very closely to the edge velocities defined in convectively unstable systems. (See in particular Ref. 13.) In this case one defines $V^{-}$and $V^{+}$to be the $x / t$ rays that separate regions of growth from regions of decay in the space-time diagram. The growth rate along rays defining the edges is zero. For the experimental data, the growth rate along rays is shown in Fig. 4(d), where the envelope of $U_{y}$, sampled along different $x / t$ rays, is plotted against ray velocity. The edge velocities $V^{+}$and $V^{-}$ obtained from inflection points are indicated by vertical dashed lines in Fig. 4(d). It can be seen that these are in excellent agreement with the zero growth-rate rays. Moreover, the group velocity $V_{g}$ (vertical solid line) is also agrees very well with the maximum of the envelop in the ray representation.

Thus it can be seen that the velocities are all quite well defined experimentally, as least up to the time at which the perturbation reaches its maximum, even though the flow is spatially inhomogeneous and the wave packet lives only a finite time. As expected none of the velocities (including $V_{p}$ ) are strictly constant over this space-time region, but they are very nearly so outside of the nearwake region. We have not attempted to extract their detailed variation in the present study.

We now consider the behavior of the different velocities as $|\epsilon| \rightarrow 0$ (i.e when $\operatorname{Re} \rightarrow \operatorname{Re}_{c}$ ). In Fig. 5 (a) we can see that the phase velocity $V_{p}$, and equivalently the leading edge velocity $V^{+}$, normalized by the inflow speed, exhibit a slight decrease with increasing Reynolds number. Generally, the value of $V_{p} / U_{0}$ in the subcritical regime $(\epsilon<0)$ compares well with the value of 0.88 reported in the literature [18] for the supercritical $(\epsilon>0)$ regime. The group and trailing edge velocities are summarized in Fig. 5(b). Both velocities decrease with increasing 

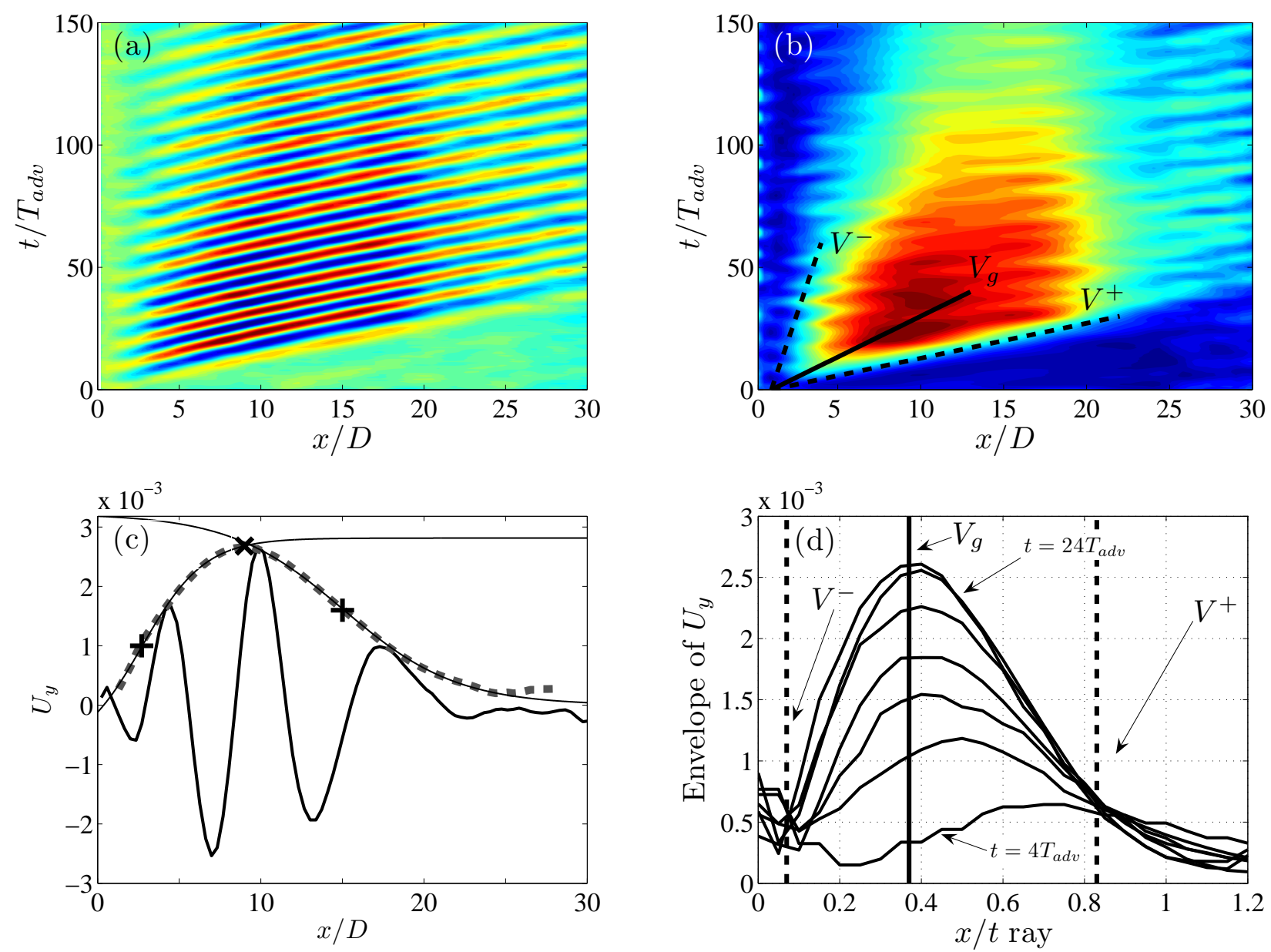

FIG. 4: Evolution of the wave packet seen in spatiotemporal diagrams for $\epsilon=-0.137$. (a) Cross-stream velocity $U_{y}(x, t)$, (b) envelope of $U_{y}(x, t),(\mathrm{c}) U_{y}(x)$ and its envelope (bold dashed curve) at a fixed time $\left(t=24 T_{a d v}\right)$. Also shown (thin curves) are tanh fits to the relevant part of the envelope. The infection points and envelope maximum are indicated. (d) Envelope of $U_{y}$ sampled along various rays as a function of ray velocity. The edge and group velocities, obtained from the inflection points and envelope maxima, are indicated with vertical lines.

Reynolds number as one would expect in approaching the global wake instability. To within experimental accuracy, $V^{-}$approaches zero as $\epsilon \rightarrow 0$.

From the spatiotemporal diagrams we are also able to pinpoint the position in space and time $\left(X_{\max }, T_{\max }\right)$ at which the subcritical response reaches maximum amplitude. See Fig. 6(a). Again we use the cross-stream velocity component $U_{y}$. We let $A_{\max }$ denote the maximum of the response, so that $A_{\max }=U_{y}\left(X_{\max }, T_{\max }\right)$. Figures 6(b) through 6(d) show the dependence of the maximum on reduced Reynolds number. It is not surprising that $A_{\max }$ grows when the global instability threshold is approached $(|\epsilon| \rightarrow 0)$, as can be seen in Fig. 6(b), because the susceptibility of the flow increases near the onset. On the contrary, the behavior of $X_{\max }$ (which diminishes when $|\epsilon| \rightarrow 0$, see Fig. 6(c), deserves a further comment, since it seems to be opposite to the case of other instabilities in the subcritical regime where the characteristic length scale increases when $|\epsilon| \rightarrow 0$ (e.g. the penetration length in pre-transitional Rayleigh-Bénard convection [19]). The fact that the maximum of the instability moves closer to the cylinder (i.e. that $X_{\max }$ diminishes) when approaching the threshold while the time at which this occurs remains constant [on a nondimensional scale normalized by the advective time scale $T_{a d v}, \mathrm{Fig}$. 6(d)] is actually consistent with $V_{g}$ decreasing as the convectively unstable system tends to the absolute instability threshold. The difference with the case of Rayleigh-Bénard convection comes from the effect of the mean flow advection, which modifies the physical meaning of the penetration lengthscale.

For a given value of $\epsilon$, an increase in the strength of the perturbation produces a response in which the maximum amplitude $A_{\max }$ is slightly larger and occurs at a position closer to the cylinder (smaller $X_{\max }$ ). Approaching the threshold reduces the effect of changing the perturbation 

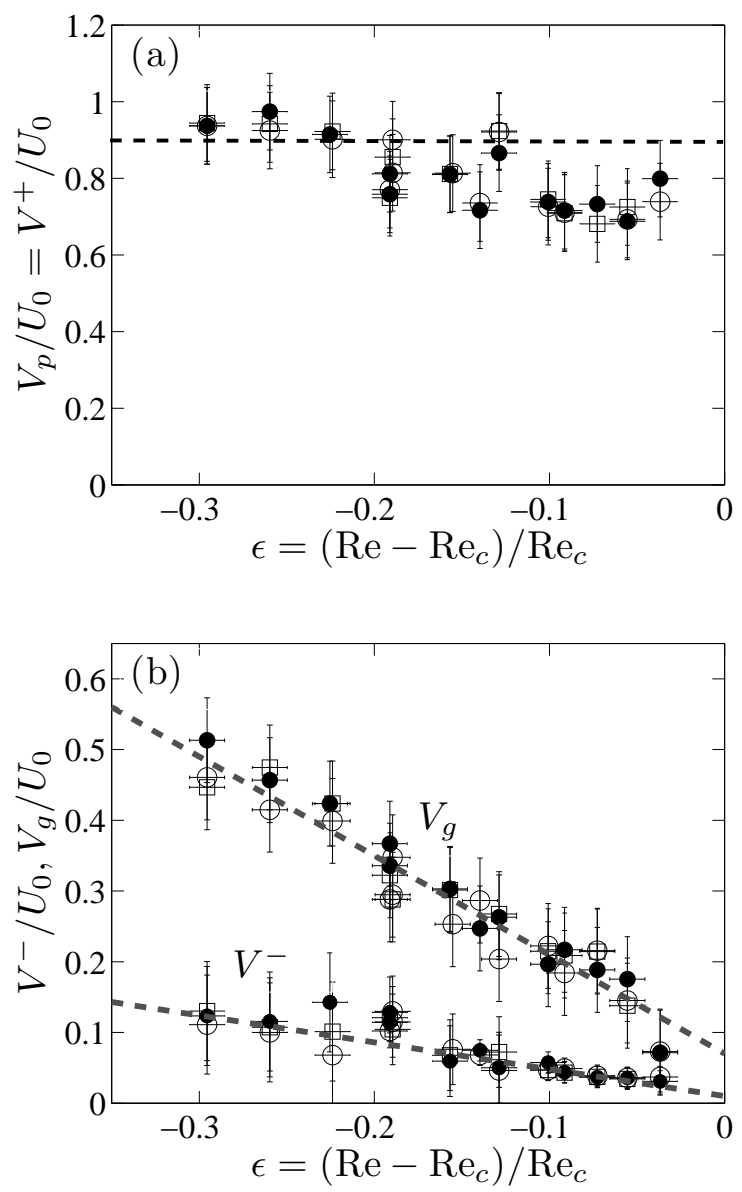

FIG. 5: (a) Phase velocity $V_{p}$ (which is also $V^{+}$) and (b) group velocity $V_{g}$ and trailing-edge velocity $V^{-}$as a function of $\epsilon$. The dashed line in (a) corresponds to the value of $V_{p} / U_{0}$ for the Bénard-von Kármán wake $\left(\operatorname{Re}>\operatorname{Re}_{c}\right)$. The dashed lines in (b) are linear fits to the $V_{g}$ and $V^{-}$data. Experimental points are labeled as $\bullet$ : small perturbation, $\circ$ : medium perturbation, $\square$ : strong perturbation. Horizontal error bars indicate the uncertainty in the Reynolds number measurement.

strength.

\section{TRANSIENT GROWTH}

As noted in the introduction, an important feature of the cylinder wake is the inhomogeneous nature of the flow. Hence, even though the subcritical response just presented has much of the general character of convective instability, perturbations do not grow indefinitely (even linearly) as they would for a homogeneous convectively unstable system. Rather, a localized initial perturbation grows at first, due to local flow features near the cylinder, but is simultaneously advected downstream into a region of stability where the perturbation decays. Hence in the absence of any inflow noise the impulsive response is only transient. Such behavior is known in inhomogeneous flows [5, 20 27]. In should be noted, however, that inflow or other noise may modify the picture in that the localized region of instability acts as an amplifier and sustained dynamics may arise in some cases even in the subcritical regime [11, 24, 27, 30, 31.

An increasingly common approach to quantifying the transient response of flows is in terms of their transient energy growth 28]. Such an analysis provides a global measure of the response dynamics. Here we undertake such an analysis of experimental data.

We define the perturbation energy from our measurements as follows:

$$
E(t)=\iint_{\Delta}\left(u_{x}^{2}+u_{y}^{2}\right) d x d y
$$

where

$$
u_{x}=U_{x}-U_{x b a s e} \quad u_{y}=U_{y}-U_{y b a s e},
$$

where the base flow $\left(U_{x b a s e}, U_{\text {ybase }}\right)$ is the measured steady flow before any perturbation.

Experimentally, the total energy of the perturbation can only be measured while the packet is contained in the observation window. Hence, the energy calculated from the velocimetry data does not include the contribution from vortices that have been advected out of the measurement area. In order to quantify this effect we compare the energy calculated using two different streamwise sizes for the integration area $\Delta$ : the total energy $E_{t o t}$, where the whole measurement window is used, and the energy of the first half of the wave packet $E_{\text {half }}$, where the downstream boundary of the integration area $\Delta$ is given by the time-dependent position of the maximum perturbation amplitude $\tilde{X}_{\max }(t)$ - see Fig. $7($ a). We note that $\tilde{X}_{\max }(t)$ is time-dependent and should not be confused with $X_{\max }$ shown in Fig 6(c). The quantities are related via: $X_{\max }=\max _{t} \tilde{X}_{\max }(t)$. We find that the ratio of $E_{\text {tot }} / E_{\text {half }}$ remains approximately constant $(\approx 2)$ in time, which means that the measurement window is sufficiently large to capture the dynamics of the perturbation growth and decay before the effect of the flow structures advected away from the downstream boundary of the measurement window becomes significant.

The time evolution of the energy is shown in Fig. 7(b). The value of $E_{t o t}$, as well as $E_{\text {half }}$ and $E_{t o t}-E_{\text {half }}$, are shown. All energies are all normalized by $E_{0}$, the value of $E_{t o t}$ at the first measured instance following the impulse. The perturbation energy initially undergoes growth until $t \approx 50 T_{a d v}$, at which point the energy decays. This is precisely the transient growth dynamics expected of convective instabilities in inhomogeneous media.

The late time behavior of the energy corresponds to the exponential decay of the least stable normal mode in a stable region, e.g. [7, 29]. As the system approaches the absolute instability threshold $(\epsilon \rightarrow 0)$ this decay becomes slower. This can be be seen in Fig. 7(c), where the decay rate is given by the slope of the curves of $\log E_{t o t}$ and 

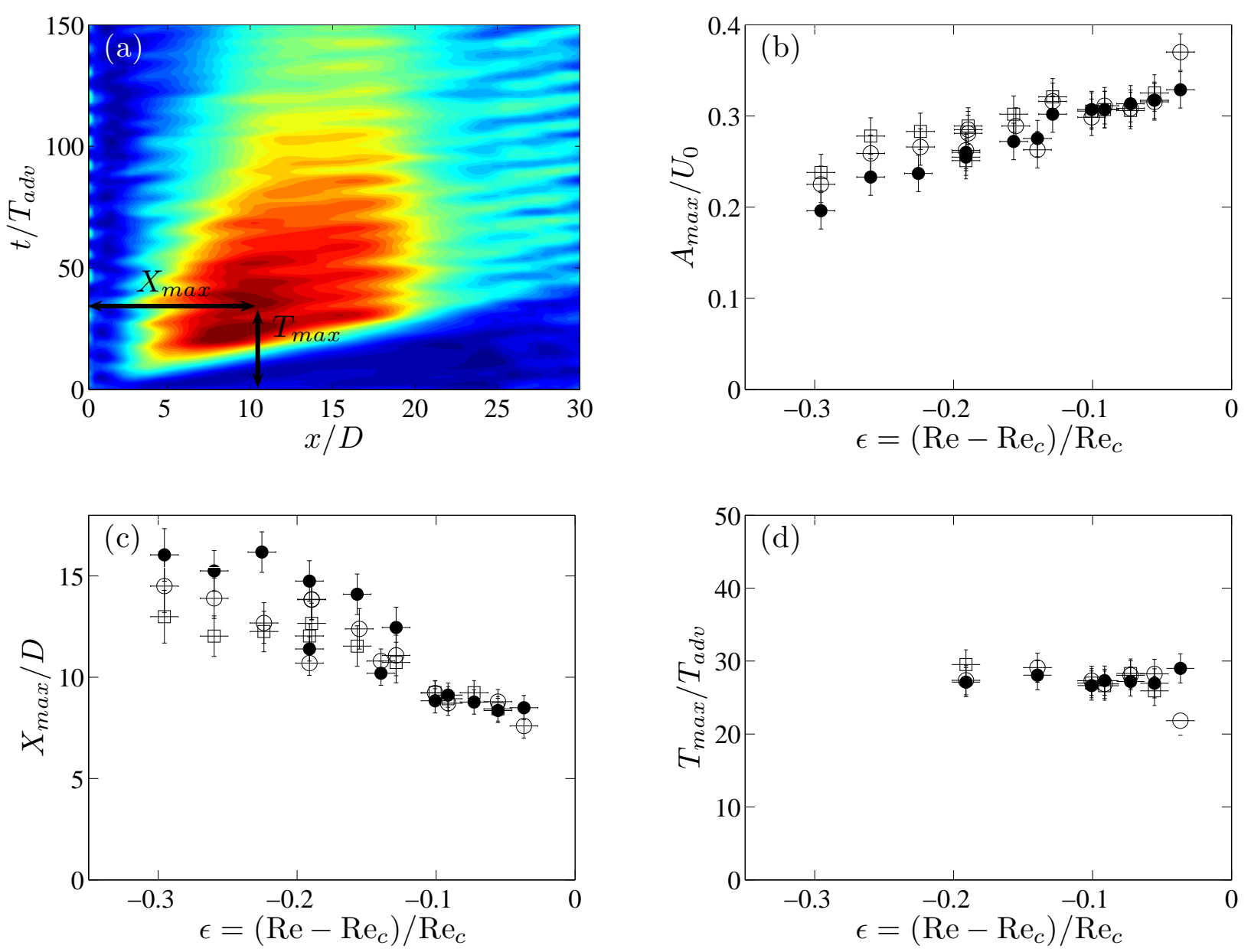

FIG. 6: (a) Definition of the maximum of the perturbation on the spatiotemporal diagram. (b) Evolution of the maximum of the perturbation $A_{\max }$, (c) $X_{\max }$ and (d) $T_{\max }$ as a function of the reduced Reynolds Number $\epsilon$. $A_{\max }, X_{\max }$ and $T_{\max }$ are rendered nondimensionalized respectively by the free stream velocity $U_{0}$, the cylinder diameter $D$ and the advective timescale $T_{a d v}=D / U_{0}$. Experimental points are labeled as $\bullet$ : low perturbation, o: medium perturbation, $\square$ : strong perturbation.

more quantitatively in Fig. $8(a)$ where the asymptotic decay rate is plotted as a function of reduced Reynolds number. For comparison, decay rates from linear stability computations [6] shown with a solid curve. The agreement is excellent.

The energy growth at short times can be examined using the maximum of the energy $E_{\max }$ and the time for which this maximum is reached $t_{\max }$ as a function of $\epsilon$. See Fig. 8(b). Consistently with the approach of the absolute instability threshold, $E_{\max }$ increases when $|\epsilon| \rightarrow 0$. The time $t_{\max }$ where the maximum energy is reached remains approximately constant.

\section{DISCUSSION AND CONCLUSIONS}

The convectively unstable wave packets produced by an impulsive perturbation in a subcritical cylinder wake have been studied experimentally. Velocity field mea- surements obtained by PIV have permitted us to characterize quantitatively the instability wave, shedding light on points that remained not clearly analyzed in the literature. Firstly, probing the perturbation of the velocity field due to the instability shows unambiguously that there is a well-defined maximum of the perturbation amplitude in the wake. This differs significantly from the picture given by the streakline visualizations usually used to illustrate the convective instability [17], which distort the observation of the actual growth and decay. The value of this maximum and its position downstream the cylinder depend on the Reynolds number and, less markedly, on the strength of the perturbation, whereas its position in time remains constant. In addition, the evolution of the wave packet has been characterized with respect to the Reynolds number using the leading and trailing fronts as well as a typical group velocity. The measured velocities are consistent with the transition from a convective to an absolute global instability as 

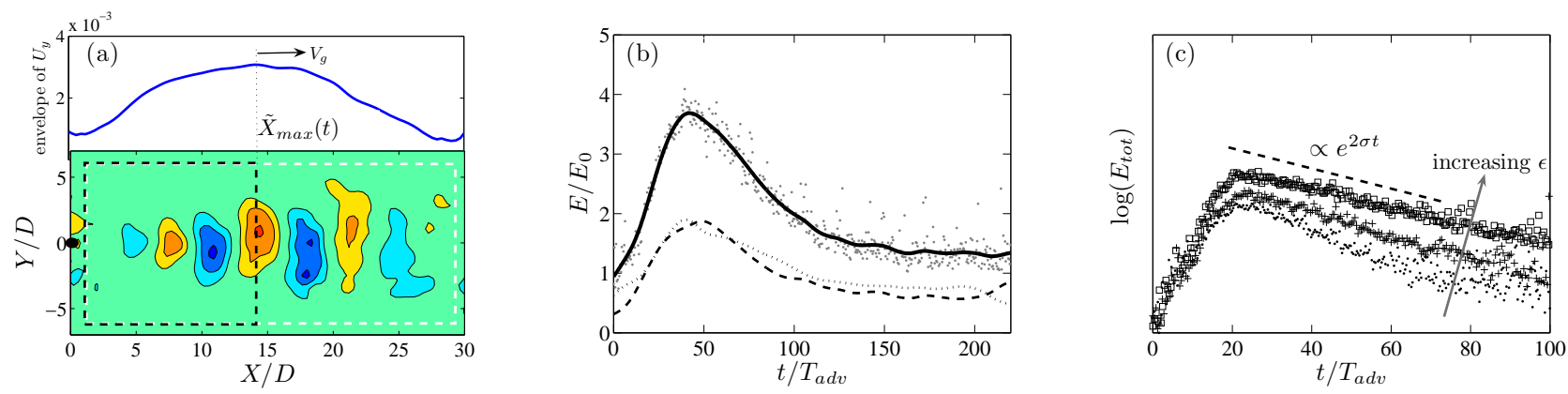

FIG. 7: Transient energy growth in experiment. (a) Illustration of the integration area for the energy on the instantaneous velocity field $U_{y} . E_{t o t}$ and $E_{\text {half }}$ are calculated over the white and black rectangles, respectively. The downstream boundary of the black rectangle moves at a speed $V_{g}$ and tracks the maximum of the perturbation envelope as indicated in the upper plot. (b) Energy as a function of time for $\epsilon=-0.14$. The dots are the experimental data of $E_{t o t}$. The solid, dashed and dotted lines are smooth fits of $E_{t o t}, E_{\text {half }}$ and $E_{t o t}-E_{\text {half }}$, respectively. (c) Time evolution of the energy on logarithmic scale, for three different values of the reduced Reynolds number $\epsilon$ : $\bullet \epsilon=-0.19,+\epsilon=-0.14$ and $\square \epsilon=-0.1$. The slope of the energy decay on the logarithmic scale gives the decay rate $\sigma$.

the Reynolds number increases towards the Bénard-von Kármán instability threshold (see Fig. (5)).

The analysis of the transient energy growth associated to the instability also deserves a final comment. The qualitative features of the temporal evolution of the energy agree with the standard picture of transient growth due to convective instability in inhomogeneous media, i.e. a short-time algebraic growth followed by an exponential decay at late times. However, the measured values of $\max \left(E_{t o t} / E_{0}\right)$ are remarkably low (always less than 10) when compared to the values obtained by numerical computations (of order $10^{3}$, see e.g. [25, 27]). The main reasons for this discrepancy are most likely the fact that in the experiment one does impose an optimal pertur- bation and also the fact that extracting $E_{0}$, the initial energy of the perturbation, is experimentally quite difficult. This discrepancy raises the question about the pertinence of the energy gain (ubiquitous parameter in transient growth studies) as the most appropriate quantity to use for comparison between theory and experiments.

\section{Acknowledgments}

DB gratefully acknowledges support from the Leverhulme Trust and the Royal Society.
[1] M. Provansal, C. Mathis, and L. Boyer, Benard-von Kármán instability: transient and forced regimes, J. Fluid Mech. 182, 1 (1987).

[2] C. P. Jackson, A finite-element study of the onset of vortex shedding in flow past variously shaped bodies, J. Fluid Mech. 182, 23 (1987).

[3] B. Noack and H. Eckelmann, A global stability analysis of the steady and periodic cylinder wake, J. Fluid Mech. 270 (1994).

[4] B. Pier, On the frequency selection of finite-amplitude vortex shedding in the cylinder wake, J. Fluid Mech. 458, 407 (2002).

[5] J.-M. Chomaz, Global instabilities in spatially developing flows: non-normality and nonlinearity, Annu. Rev. Fluid Mech. 37, 357 (2005).

[6] D. Barkley, Linear analysis of the cylinder wake mean flow, Europhysics Lett. 75, 750 (2006).

[7] F. Giannetti and P. Luchini, Structural sensitivity of the first instability of the cylinder wake, J. Fluid Mech. 581, 167 (2007).

[8] L. D. Landau and E. M. Lifshitz, Fluid Mechanics, Pergamon Press, London, 1959.
[9] P. Huerre and P. A. Monkewitz, Absolute and convective instabilities in free shear layers, J. Fluid Mech. 159, 151 (1985).

[10] P. Huerre and P. A. Monkewitz, Local and global instabilities in spatially developing flows, Annu. Rev. Fluid Mech. 22, 473 (1990).

[11] R. J. Deissler, Noise-sustained structure, intermittency, and the ginzburg-landau equation, J. Stat. Phys. 40, 371 (1985).

[12] W. V. Saarloos, Front propagation into unstable states, Phys. Rep. 29 (2003).

[13] I. Delbende and J. Chomaz, Nonlinear convective/absolute instabilities in parallel two-dimensional wakes, Phys. Fluids 10, 2724 (1998).

[14] P. A. Monkewitz, The absolute and convective nature of instability in two-dimensional wakes at low reynolds numbers, Phys. Fluids 31, 999 (1988).

[15] J. A. Zielinska and J. E. Wesfreid, On the spatial structure of global modes in wake flow, Phys. Fluids 7 (1995).

[16] M.F. Schatz, D. Barkley, and H.L. Swinney, Instabilities in spatially periodic channel flow, Phys. Fluids 7, 344 (1995). 

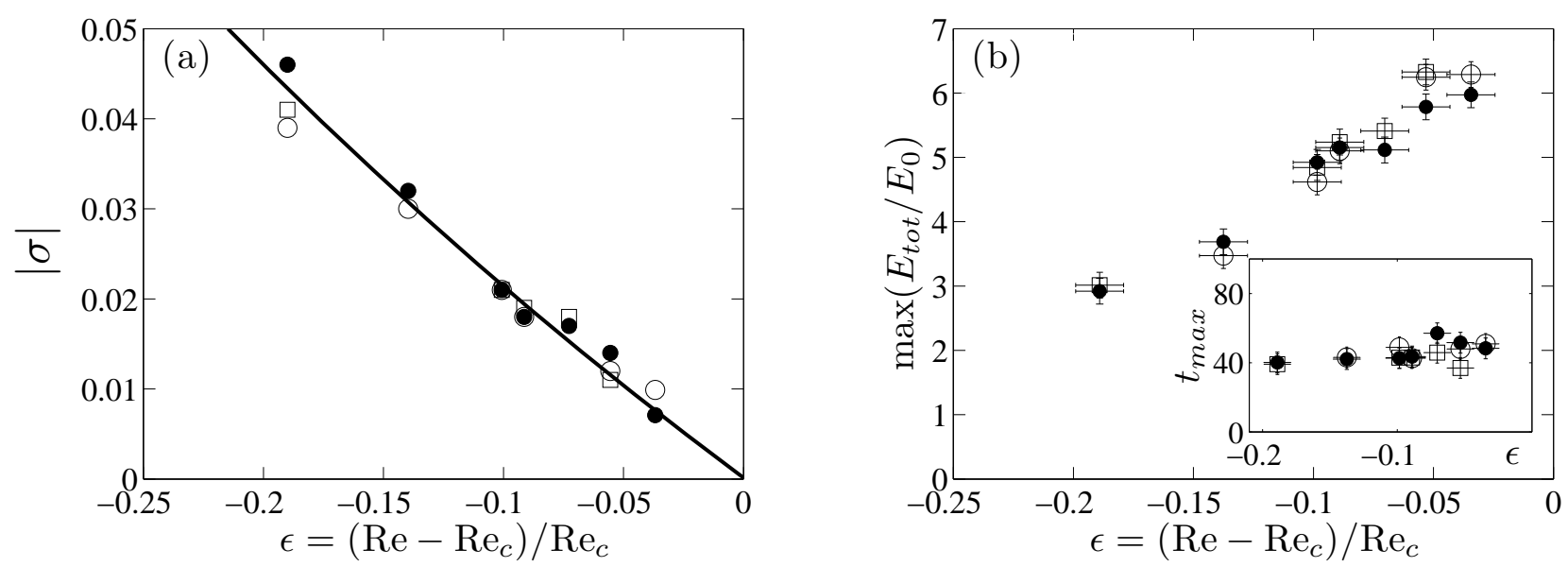

FIG. 8: (a) Asymptotic decay rate of perturbations [as seen in Fig. [7(c)] as a function of reduced Reynolds number $\epsilon$. Experimental points are labeled as $\bullet$ : low perturbation, $\circ$ : medium perturbation, $\square$ : strong perturbation. The curve shows decay rates from linear stability computations. (b) Maximum transient energy gain and (inset) time at which this maximum occurs as, a function of the reduced Reynolds number $\epsilon$. Experimental points are labeled as • : low perturbation, $\circ$ : medium perturbation, $\square$ : strong perturbation.

[17] P. L. Gal and V. Croquette, Visualization of the spacetime impulse response of the subcritical wake of a cylinder, Phys. Rev. E 62 (2000).

[18] C. Williamson, Oblique and parallel modes of vortex shedding in the wake of a circular cylinder at low reynolds numbers, J. Fluid Mech. 206, 579 (1989).

[19] J. Wesfreid, P. Bergé, and M. Dubois, Induced pretransitional Rayleigh-Bénard convection, Phys. Rev. A 19, 1231 (1979).

[20] C. Cossu and J. M. Chomaz, Global measures of local convective instabilities, Phys. Rev. Lett. 78, 4387 (1997).

[21] P. Gondret, P. Ern, L. Meignin, and M. Rabaud, Experimental evidence of a nonlinear transition from convective to absolute instability, Phys. Rev. Lett. 82, 1442 (1999).

[22] L. Kim and J. Moehlis, Transient growth for streakstreamwise vortex interactions, Phys. Lett. A 358, 431437 (2006).

[23] H. Blackburn, D. Barkley, and S. Sherwin, Convective instability and transient growth in flow over a backwardfacing step, J. Fluid Mech. 603, 271 (2008).

[24] O. Marquet, D. Sipp, J.-M. Chomaz, and L. Jacquin, Amplifier and resonator dynamics of a low-Reynoldsnumber recirculation bubble in a global framework, J. Fluid Mech. 605, 429 (2008).
[25] N. Abdessemed, A. S. Sharma, S. J. Sherwin, and V. Theofilis, Transient growth analysis of the flow past a circular cylinder, Phys. Fluids 21, 044103 (2009).

[26] C. D. Cantwell, D. Barkley, and H. Blackburn, Transient growth analysis of flow through a sudden expansion in a circular pipe, Phys. Fluids 22, 034101 (2010).

[27] C. D. Cantwell and D. Barkley, Computational study of subcritical response in flow past a circular cylinder, Phys. Rev. E 82, 026315 (2010).

[28] P. J. Schmid and D. S. Henningson, Stability and Transition in Shear Flows, Springer, 2001.

[29] R. H. Hernández and A. Pacheco, Numerical simulation and experiments of a control method to suppress the Bénard von Kármán instability, Eur. Phys. J. B 30, 265 (2002).

[30] G. Gauthier, P. Gondret, and M. Rabaud, Axisymmetric propagating vortices in the flow between a stationary and a rotating disk enclosed by a cylinder, J. Fluid Mech. 386, 105 (1999).

[31] J.M. Lopez, F. Marques, A.M. Rubio, and M. Avila, Crossflow instability of finite Bödewadt flows: Transients and spiral waves, Phys. Fluids 21, 114107 (2009). 